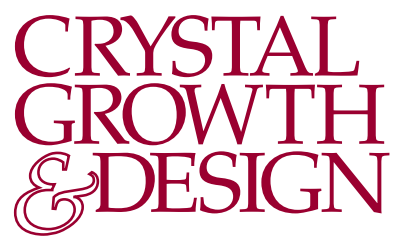

\title{
Effect of Sn Addition on Epitaxial GaAs Nanowire Grown at Different Temperatures in Metal-Organic Chemical Vapor Deposition
}

\author{
Han Gao, ${ }^{\dagger} \odot$ Qiang Sun, ${ }^{\dagger}$ Mykhaylo Lysevych, ${ }^{\S}$ Hark Hoe Tan,, Chennupati Jagadish," \\ and Jin Zou, ${ }^{*}, * \odot$
}

${ }^{\dagger}$ Materials Engineering, ${ }^{\ddagger}$ Centre for Microscopy and Microanalysis, The University of Queensland, St. Lucia, Queensland 4072,
Australia
${ }^{\S}$ The Australian National Fabrication Facility, "Department of Electronic Materials Engineering, Research School of Physics and
Engineering, The Australian National University, Canberra, Australian Capital Territory 2601, Australia

ABSTRACT: In this study, we investigated the growth behaviors of GaAs nanowires with tetraethyl-tin $(\mathrm{Sn})$ as addition grown at different temperatures in a metal-organic chemical vapor deposition system. It was found that the nanowire axial growth rate can be influenced by the addition of $\mathrm{Sn}$ in opposite ways at different growth temperatures. The growth rate of nanowires is higher because of the enhanced decomposition of trimethyl gallium (TMGa) with increasing the $\mathrm{Sn}$ addition at $390{ }^{\circ} \mathrm{C}$ while lower because of the lower

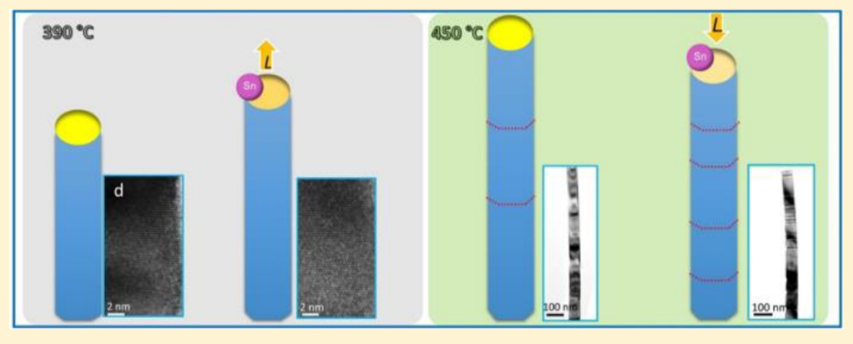
catalyst supersaturation level with increasing the $\mathrm{Sn}$ addition at $450{ }^{\circ} \mathrm{C}$. With the $\mathrm{Sn}$ addition, nanowire quality can be maintained at $390{ }^{\circ} \mathrm{C}$ because the lower temperature benefits stabilizing the structure but further degraded at $450{ }^{\circ} \mathrm{C}$ when compared with intrinsic nanowires. This study provides an insight into the effect of the Sn addition on GaAs nanowire growth, which will be useful for the design of nanowire-based devices.

\section{INTRODUCTION}

III-V semiconductor nanowires have been considered as promising one-dimensional nanoscale materials in nanoelectronics and optoelectronic applications due to their high aspect ratio and unique electrical and optical properties. ${ }^{1-3}$ For practical device applications in using nanowires as building blocks, it is necessary to understand their growth mechanism so that the required structural, physiochemical, electrical, and optical properties can be controlled. As a kind of representative III-V semiconductor nanowires, GaAs nanowires have received great attention due to their promising applications in solar cells, ${ }^{4,5}$ nanolasers, ${ }^{6}$ and the next-generation transistors. ${ }^{7}$ Indeed, their epitaxial growth using the metalcatalyzed approach has been well developed. ${ }^{8}$ In many applications, however, the electrical properties of the nanowires need to be controlled by means of doping. ${ }^{9}$ So far, less effort has been devoted to the epitaxial growth of doped III-V semiconductor nanowires. ${ }^{10-14}$ Although examples such as ptype doping in GaAs nanowires using $\mathrm{C}^{15} / \mathrm{Zn}^{16,17} / \mathrm{Be}^{18} \mathrm{n}$-type doping in $\mathrm{GaAs}$ nanowires using $\mathrm{Si}^{19} / \mathrm{Te}^{20} / \mathrm{Sn}^{21,22}$ dopants have been reported, the growth behaviors of doped nanowires are not fully understood as these impurities may cause undesirable effects. For instance, using dimethyl-zinc as the $\mathrm{Zn}$ dopant precursor, axial growth of InP nanowires can be significantly enhanced ${ }^{24}$ while the axial growth of InAs nanowires is impeded with the $\mathrm{Si}$ addition. ${ }^{23}$ In addition, impurities may also change the crystal structure s-27 $^{25}$ and structural quality $^{28,29}$ of the nanowires. The $\mathrm{Zn}$ addition has modified the catalyst-nanowire interfacial energy and hence the contact angles, leading to the switch of the InP nanowires from the zinc blende (ZB) structure to a wurtzite (WZ) structure. $^{25}$ Thus the properties of doped nanowires are not only affected by the incorporated dopants but can also be strongly influenced by the change in their morphology and crystal structure. $^{30-32}$ Therefore, it is necessary to clarify the mechanism of how impurities influence nanowire growth.

In this study, we investigate the effect of tetraethyl-tin (TESn) on the epitaxial growth of GaAs nanowires grown via the metal-catalyzed approach at different growth temperatures in a metal-organic chemical vapor deposition (MOCVD) system. It is found that the TESn addition has an opposite effect on the axial growth rate of GaAs nanowires grown at different temperatures. Specifically, with increasing the TESn addition, the axial growth rate increases only marginally when GaAs nanowires grown at $390{ }^{\circ} \mathrm{C}$ but decreases significantly at $450{ }^{\circ} \mathrm{C}$ when compared with the growth of undoped $\mathrm{GaAs}$ nanowires. The structural quality of nanowires grown at 450 ${ }^{\circ} \mathrm{C}$ is also severely degraded with the TESn addition. Through detailed electron microscopy characterization, the effects of the TESn addition on the growth mechanism of GaAs nanowire are clarified and discussed.

Received: June 17, 2019

Revised: July 11, 2019

Published: July 18, 2019 


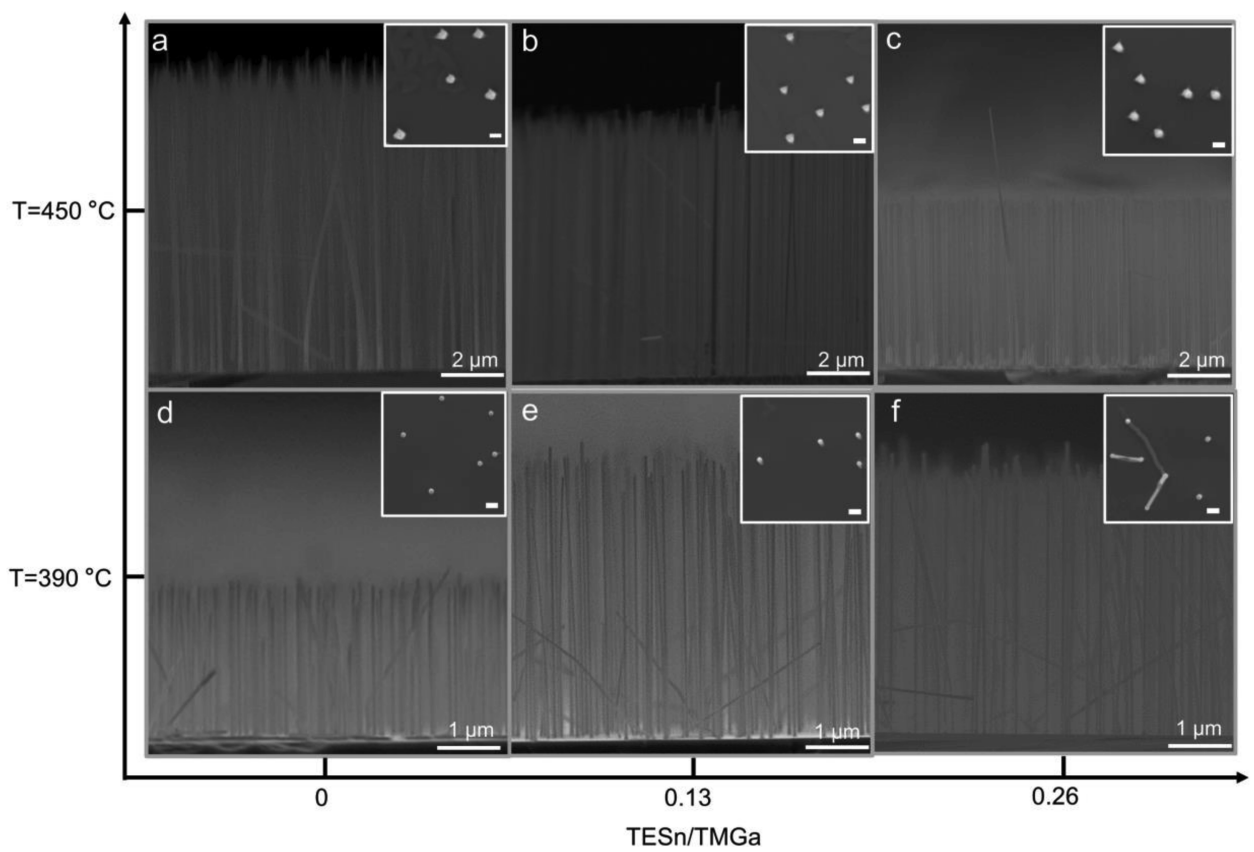

Figure 1. SEM side views of as-grown nanowires on substrates. $(\mathrm{a}-\mathrm{c})$ Nanowires grown at $450{ }^{\circ} \mathrm{C}$ with TESn addition of (a) 0 (reference), (b) $\mathrm{TESn} / \mathrm{TMGa}=0.13$, and $(\mathrm{c}) \mathrm{TESn} / \mathrm{TMGa}=0.26 .(\mathrm{d}-\mathrm{f})$ Nanowires grown at $390^{\circ} \mathrm{C}$ with TESn of $(\mathrm{d}) 0$ (reference), (e) TESn/TMGa $=0.13$, and (f) TESn/TMGa $=0.26$. Insets show the top views on substrate and nanowires. Scale bars in insets are $200 \mathrm{~nm}$.

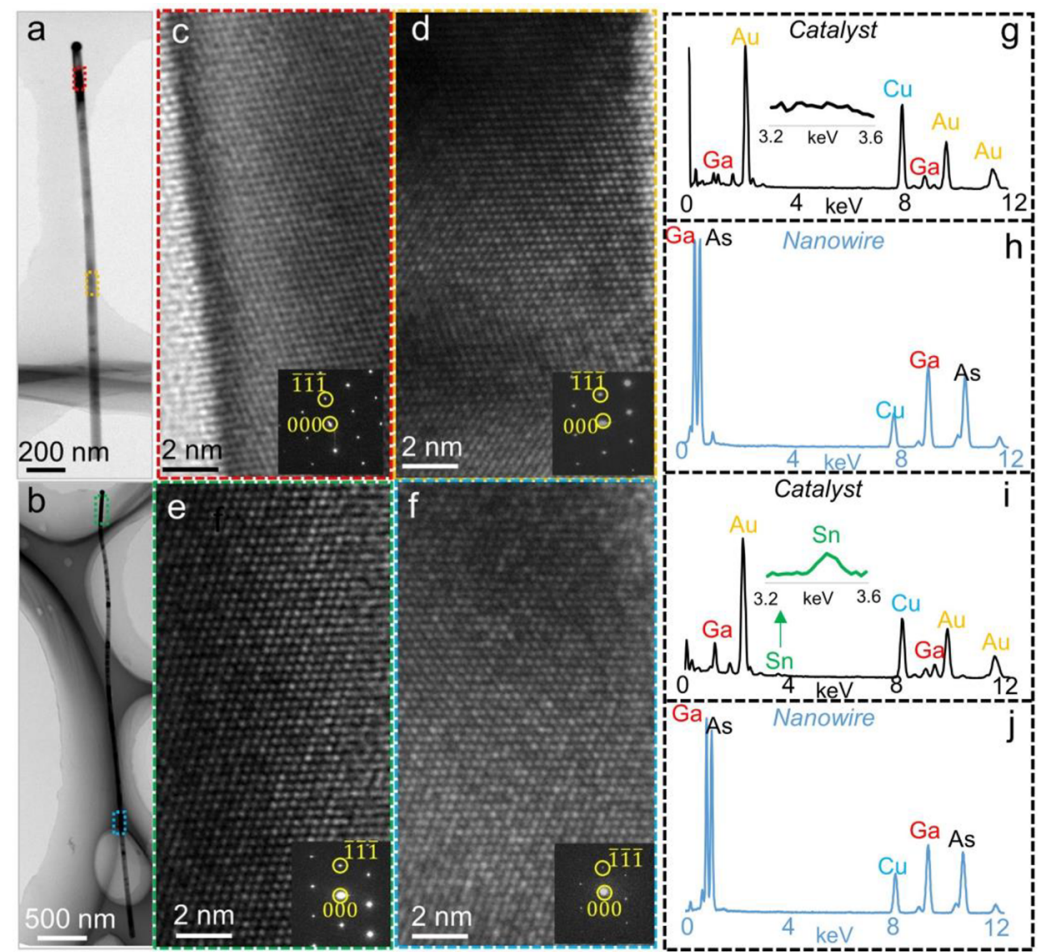

Figure 2. TEM images of typical reference GaAs nanowire (without TESn, a) and the GaAs nanowire with TESn addition (TESn/TMGa $=0.26$, b) grown at $390{ }^{\circ} \mathrm{C}$. (c,d and e,f) HRTEM images and corresponding SAED patterns of the marked regions in (a) and (b), respectively. Typical EDS results on nanowires compositions without TESn $(\mathrm{g}-\mathrm{h})$ and with TESn addition $(\mathrm{i}-\mathrm{j})$. Insets in $(\mathrm{g})$ and $(\mathrm{i})$ show the $10 \times$ magnified EDS spectra between $3.2-3.6 \mathrm{keV}$.

\section{EXPERIMENTAL SECTION}

Epitaxial nanowire growths were performed in an AIXTRON 200/4 horizontal flow MOCVD system. The chamber pressure was maintained at 100 mbar with total gas flow rate at 15 standard liters per minute. Trimethyl-gallium (TMGa), arsine $\left(\mathrm{AsH}_{3}\right)$, and TESn were used as group III, V, and IV precursors, respectively, carried by ultrahigh-purity hydrogen gas. Prior to nanowire growth, GaAs $(\overline{111})$ substrates were cleaned by deionized water and pretreated by poly-Llysine (PLL). Then $50 \mathrm{~nm}$ diameter colloidal Au particles (British Biocell International-Ted Pella, Inc.) were dispersed and adhered onto substrate surfaces. The substrates were then annealing at $600{ }^{\circ} \mathrm{C}$ under $\mathrm{AsH}_{3}$ ambient for $10 \mathrm{~min}$ in the MOCVD reaction chamber to 


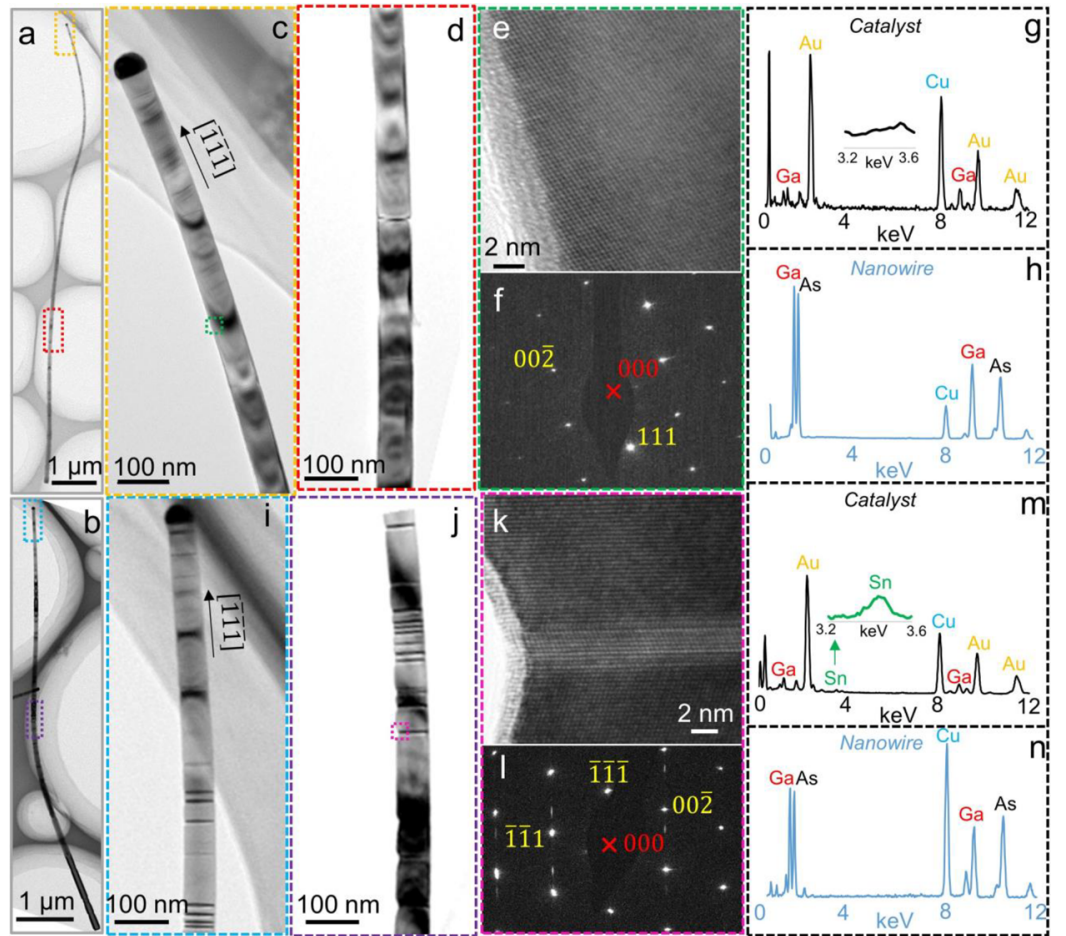

Figure 3. TEM investigation on a typical reference GaAs nanowire and GaAs nanowire with high TESn addition grown at $450{ }^{\circ} \mathrm{C}$. (a-b) BF-TEM images for individual nanowires. ( $c-d$ and $i-j)$ Enlarged areas in (a) and (b). (e-f and $k-1)$ HRTEM images and corresponding SAED patterns with index of the marked areas in $(c)$ and $(j)$ respectively. $(g-h$ and $m-n)$ Typical EDS results show the composition of catalysts and nanowires. Insets in $(\mathrm{g})$ and $(\mathrm{m})$ show the $10 \times$ magnified EDS spectra between $3.2-3.6 \mathrm{keV}$.

remove oxide contaminations from the substrate surfaces. After cooling to $450{ }^{\circ} \mathrm{C}$, nanowire nucleation was performed for 1 min by switching on TMGa. The reaction chamber was then set to the nanowire growth temperature of 450 and $390{ }^{\circ} \mathrm{C}$, followed by $\sim 30$ min nanowire growth with switched on the TESn addition. During nanowire growth, flux rates of TMGa and $\mathrm{AsH}_{3}$ were set at $1.157 \times$ $10^{-5}$ and $5.357 \times 10^{-4} \mathrm{~mol} / \mathrm{min}$, respectively, which gave a $\mathrm{V} / \mathrm{III}$ ratio of 46 for all nanowire growths. Three different TESn flow rates were used, in the range from 0 to $2.993 \times 10^{-6} \mathrm{~mol} / \mathrm{min}$, giving a nominal $\mathrm{Sn} / \mathrm{Ga}$ ratio as $0,0.13$ and 0.26 , respectively. Growth was terminated by switching off the TMGa and TESn sources simultaneously and cooled down in the presence of $\mathrm{AsH}_{3}$ until $350{ }^{\circ} \mathrm{C}$.

The nanowire morphology was determined by scanning electron microscopy (SEM, JEOL Field-Emission 7800, operated at $5 \mathrm{kV}$ ), and their structural and compositional characteristics were investigated by transmission electron microscopy (TEM, FEI Tecnai F20, $200 \mathrm{kV}$, equipped with energy dispersive X-ray spectroscopy, EDS). SEM specimens were prepared by cleaving the GaAs substrates along the $\{110\}$ atomic planes (note that nanowires still attach on the substrates). Individual nanowires for TEM investigations were prepared through ultrasonicating the substrates in ethanol and dispersing the suspended nanowires onto holey carbon-lined TEM copper grids.

\section{RESULTS AND DISCUSSION}

Figure 1 is a set of SEM images showing the side view of the nanowires grown with different TESn additions at $450{ }^{\circ} \mathrm{C}$ Figure $1 \mathrm{a}-\mathrm{c}$ and $390{ }^{\circ} \mathrm{C}$ Figure $1 \mathrm{~d}-\mathrm{f}$, respectively. The insets on the top-right corners of Figure 1 are the corresponding plan view of as-grown nanowires, confirming that nanowires grew perpendicularly on the substrate. Therefore, the nanowire lengths can be determined from their side views, from which nanowires are much longer when they grew at $450{ }^{\circ} \mathrm{C}$ (note the difference of scale bars in parts $a-c$ and $d-f$ of Figure 1 ), which can be explained by the lower energy barrier and the enhanced surface diffusivity of group III adatoms at higher temperature. ${ }^{33,34}$ The differences in nanowire lengths grown at the same temperature can be attributed to the TESn addition. For nanowires grown at $450{ }^{\circ} \mathrm{C}$, the nanowire length is reduced with the TESn addition (comparing parts $a$ and $b$ of Figure 1). With further increasing the TESn flow rate, the nanowire length reduces further (comparing parts $b$ and $c$ of Figures 1). Interestingly, an opposite trend is observed at the growth temperature of $390{ }^{\circ} \mathrm{C}$, in which the TESn addition promotes axial growth rate and therefore longer nanowires are seen (comparing parts $\mathrm{d}$ and e of Figure 1). With further increasing the TESn addition, the average length of nanowires remains similar, indicating that after a certain amount of the TESn addition, further increment in TESn does not promote the growth rate.

To understand the structural characteristics of as-grown nanowires, TEM characterization was performed. Parts $a$ and $b$ of Figure 2 show two typical nanowires grown at $390{ }^{\circ} \mathrm{C}$ without and with the highest TESn addition, respectively. Parts $c$ and $d$ of Figure 2 are high-resolution (HR) TEM images taken from the two marked regions in Figure 2a, viewed along the $\langle 110\rangle$ direction. The nanowire grown without TESn is defect-free and has a $\mathrm{ZB}$ crystal structure as shown by the HRTEM images with corresponding SAED patterns in the insets. Parts e and $f$ of Figure 2 with insets are HRTEM images and SAED patterns taken from the marked regions in Figure $2 b$, viewed along the $\langle 110\rangle$ zone axis. Similarly, when the TESn addition is used, the grown nanowires are maintained defect-free with the ZB structure. This indicates that the TESn addition does not destabilize the nanowire structural quality. Parts $g$ and h of Figure 2 show respectively the EDS spectra taken from the catalyst and the nanowire segment for the nanowires grown without the TESn addition, indicating that 


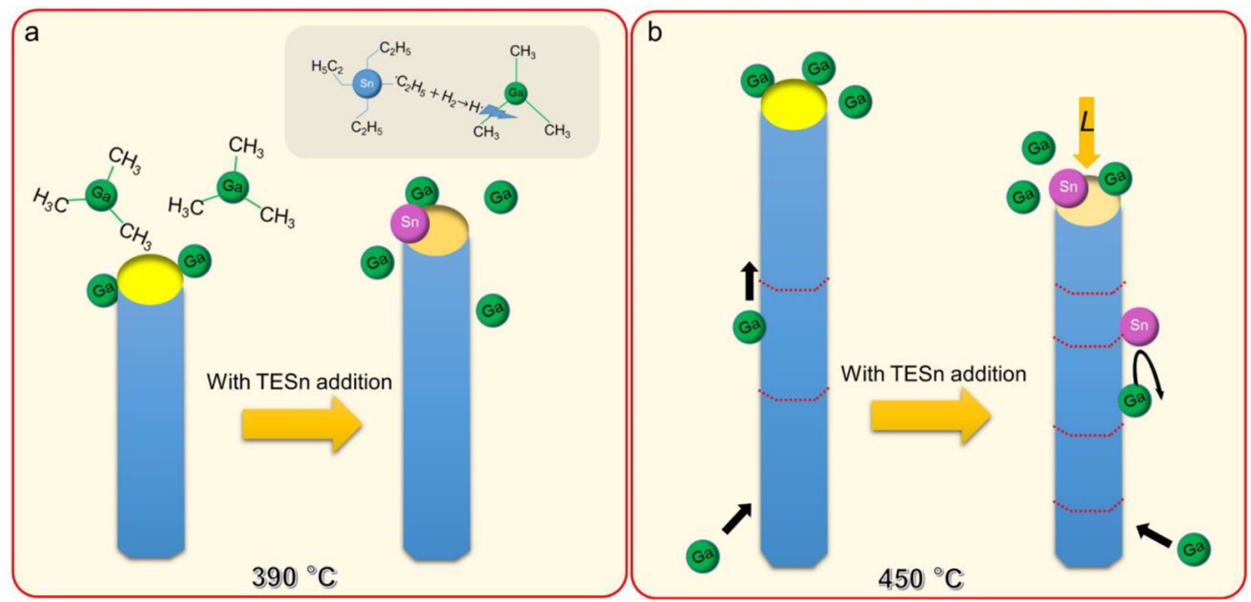

Figure 4. Schematic of GaAs nanowire growth with TESn at (a) $390{ }^{\circ} \mathrm{C}$ and (b) $450{ }^{\circ} \mathrm{C}$.

the catalyst is an $\mathrm{Au}-\mathrm{Ga}$ alloy and the nanowire is GaAs (note that $\mathrm{Cu}$ peaks are caused by the $\mathrm{Cu}$ grids). On the other hand, parts $i$ and $j$ of Figure 2 are the corresponding EDS spectra taken from the nanowire grown with the TESn addition, in which a small amount of $\operatorname{Sn}(\sim 2$ at. \%) can be detected in the catalyst but was not found in the nanowire, indicating that, if there exists a small amount of $\mathrm{Sn}$ in nanowires, it should be beyond the detectability of the EDS technique.

Similar TEM characterization was performed for the nanowires grown at $450{ }^{\circ} \mathrm{C}$. Parts $\mathrm{a}$ and $\mathrm{b}$ of Figure 3 are BF-TEM images of typical nanowires grown at $450{ }^{\circ} \mathrm{C}$ without and with the highest TESn addition, respectively. Parts $c$ and $d$ of Figures 3 and parts $i$ and $j$ of Figure 3 are magnified BFTEM images of the marked regions in parts $a$ and $b$ of Figure 3 , respectively. On the basis of the diffraction contrast in BFTEM images, both nanowires contain defects, but a higher density of defects can be witnessed in nanowires grown with the TESn addition. Parts $\mathrm{k}$ and $\mathrm{l}$ of Figure 3 are the HRTEM image and SAED pattern taken from marked area in Figure 3j, in which a ZB structure with twins can be seen. The side facets shown in Figure $3 \mathrm{k}$ can be indexed as the $\{111\}$ planes, attributed to the rotational twins. ${ }^{29,35}$ On the basis of our extensive TEM investigations of over a dozen nanowires from each specimen grown at $450{ }^{\circ} \mathrm{C}$, the average defect density of nanowires with the highest TESn addition can be estimated to be $24 \mu \mathrm{m}^{-1}$, approximately 6 times higher than that of nanowires grown without the TESn addition. Parts $g$ and $h$ and parts $m$ and $n$ of Figure 3 are EDS spectra taken, respectively, from nanowires grown without and with the TESn addition. As can be seen, like the case of nanowires grown at $390{ }^{\circ} \mathrm{C}$, Sn cannot be detected in the nanowire grown at $450{ }^{\circ} \mathrm{C}$, suggesting that the $\mathrm{Sn}$ concentration must be at a very low level below the detectable limitation of the EDS ( 1000 ppm) in our nanowires. However, the $\mathrm{Sn}$ concentration in the catalyst can be estimated as $\sim 3.5$ at. \%, nearly double the $\mathrm{Sn}$ concentration found in the catalysts of nanowires grown at 390 ${ }^{\circ} \mathrm{C}$.

On the basis of our SEM investigations, it is found that, with the TESn addition, the nanowire length increases when grown at $390{ }^{\circ} \mathrm{C}$ but decreases at $450{ }^{\circ} \mathrm{C}$ when compared with their intrinsic counterparts. Furthermore, for nanowires grown at $390{ }^{\circ} \mathrm{C}$, the growth rate increases with the TESn addition but saturated when increasing the TESn addition. To understand this phenomenon, we note that the decomposition rates of reaction precursors can influence the epitaxial growth of nanostructures in MOCVD, ${ }^{8,36}$ and temperature plays a crucial role in the decomposition of precursors. At $390{ }^{\circ} \mathrm{C}$, TMGa is not fully pyrolyzed, ${ }^{37}$ but the pyrolysis of TESn is nearly complete. ${ }^{38}$ On the other hand, it has been reported that ethyl radicals (from dopant precursors) may react with $\mathrm{H}_{2}$ (the carrier gas) and create $\mathrm{H}$-radicals, which increases the pyrolysis rate of $\mathrm{TMGa}^{26,39,40}$ so that the growth rate of nanowires is enhanced by the enriched Ga source because of the TESn addition. On the other hand, further increase of the TESn addition may hinder the Ga incorporation into catalyst ${ }^{41,42}$ and hence causes the growth rate saturating at the higher TESn addition, evidence shown in Figure 1f. Interestingly, for nanowires grown at $450{ }^{\circ} \mathrm{C}$, the TESn addition reduces the growth rate. To understand this, we note that the catalyst plays a crucial role in nanowires growth, ${ }^{25,43,44}$ and the chemical potential difference between the catalyst and underlying nanowire (the supersaturation in the catalyst) is the key driving force for axial growth. ${ }^{45}$ In our previous study, ${ }^{46}$ we found that when $\mathrm{Sn}$ is incorporated into the Au catalysts, it competes with $\mathrm{Ga}$ to form $\mathrm{Au}-\mathrm{Ga}-\mathrm{Sn}$ alloy droplets, resulting in a reduced $\mathrm{Ga}$ concentration in the catalysts and in turn lowers the catalyst supersaturation level, causing lowered nanowire growth rate. The growth rate reduction increases with increasing the TESn. In addition, it has been reported that $\mathrm{Sn}$ adatoms may act as surfactant and hinder Ga diffusion along the nanowire sidewalls, limiting the Ga source for the nanowire axial growth. ${ }^{41,47-49}$

On the basis of our TEM investigations, we found that the $\mathrm{GaAs}$ nanowires grown at $450{ }^{\circ} \mathrm{C}$ contain more defects with the TESn addition. In fact, such a phenomenon has been observed and explained in our previous study. ${ }^{46}$ Interestingly, we found that the high crystal quality of as-grown GaAs nanowires at $390{ }^{\circ} \mathrm{C}$ is still maintained, irrespective of the TESn addition, although $390{ }^{\circ} \mathrm{C}$ has been confirmed as the temperature to grow defect-free GaAs nanowires. ${ }^{50}$ To understand why the TESn addition does not influence the structural quality of nanowires grown at $390{ }^{\circ} \mathrm{C}$, we note that the low-temperature nanowire growth may benefit for stable formation of $\mathrm{ZB}$ against the instability caused by TESn addition in this study. ${ }^{51,52}$ In addition, a low growth temperature of $390{ }^{\circ} \mathrm{C}$ leads to a lower $\mathrm{Sn}$ concentration in catalysts compared with $450{ }^{\circ} \mathrm{C}$, suggesting the weaker impact of $\mathrm{Sn}$ addition on the nanowire growth front at $390{ }^{\circ} \mathrm{C} .{ }^{53,54}$ 
Consequently, the nanowire quality is maintained when the nanowire growth is at $390{ }^{\circ} \mathrm{C}$ even with the TESn addition.

To summarize the impacts of the TESn addition on GaAs nanowire growth, Figure 4 illustrates how the different functions of TESn performs on nanowire growth respectively at 390 and $450{ }^{\circ} \mathrm{C}$. Figure $4 \mathrm{a}$ shows the nanowire growth at $390{ }^{\circ} \mathrm{C}$, in which the extra H-radicals caused by the TESn addition enhance the bond breaking between $\mathrm{Ga}$ and ethyl. Therefore, the enhanced TMGa decomposition promotes the nanowire axial growth under the III-limited ambient. Figure $4 \mathrm{~b}$ illustrates the nanowire growth at $450{ }^{\circ} \mathrm{C}$, denoting that $\mathrm{Sn}$ atoms incorporate into catalyst as well as act as surfactant on side walls, which results in slowly grown nanowires. Also, the TESn addition induces higher density of defects in the nanowires when they are grown at high temperature, indicated by red dashed lines. Note that As is not taken into this schematic illustration because the growth is proceeded in Asrich ambient, and hence As is not the limiting factor in the nanowire growth.

\section{CONCLUSIONS}

In this study, we investigate the influence of the TESn addition on GaAs nanowire growth at two temperatures (namely 390 and $450{ }^{\circ} \mathrm{C}$ ) in MOCVD. Through detailed electron microscopy characterization, it has been found that, when comparing with intrinsic GaAs nanowire growth, the TESn addition causes nanowires grown longer at $390{ }^{\circ} \mathrm{C}$ but shorter at $450{ }^{\circ} \mathrm{C}$. We demonstrate that the TESn addition can promote the TMGa decomposition during the nanowire growth at $390{ }^{\circ} \mathrm{C}$, while $\mathrm{Sn}$ atoms lower the catalyst supersaturation level and may act as surfactants on the nanowire sidewalls when grown at $450{ }^{\circ} \mathrm{C}$. In addition, by benefitting from the low growth temperature, the nanowire quality can be maintained even with the $\mathrm{Sn}$ addition at $390{ }^{\circ} \mathrm{C}$. This study verifies the temperature-dependent effect of impurity addition on the epitaxial growth of GaAs nanowires, which will benefit for the design of nanowire-based device with impurity addition.

\section{AUTHOR INFORMATION}

\section{Corresponding Author}

*Phone: 61-7-33463195. E-mail: j.zou@uq.edu.au.

\section{ORCID}

Han Gao: 0000-0002-0954-0885

Qiang Sun: 0000-0001-5328-7605

Jin Zou: 0000-0001-9435-8043

\section{Notes}

The authors declare no competing financial interest.

\section{ACKNOWLEDGMENTS}

This work is financially supported by the Australian Research Council. MICROSCOPY AUSTRALIA is acknowledged for providing characterization facilities. The Australian National Fabrication Facility, ACT Node, is acknowledged for providing access to the epitaxial growth facility.

\section{REFERENCES}

(1) Lu, W.; Lieber, C. M. Nanoelectronics from the bottom up. Nat. Mater. 2007, 6 (11), 841-850.

(2) Chu, S.; Cui, Y.; Liu, N. The path towards sustainable energy. Nat. Mater. 2017, 16 (1), 16-22.
(3) Xia, Y.; Yang, P.; Sun, Y.; Wu, Y.; Mayers, B.; Gates, B.; Yin, Y.; Kim, F.; Yan, H. One-Dimensional Nanostructures: Synthesis, Characterization, and Applications. Adv. Mater. 2003, 15 (5), 353389.

(4) Li, Z.; Tan, H. H.; Jagadish, C.; Fu, L. III-V Semiconductor Single Nanowire Solar Cells: A Review. Advanced Materials Technologies 2018, 3, 1800005.

(5) Yao, M.; Huang, N.; Cong, S.; Chi, C.-Y.; Seyedi, M. A.; Lin, Y.T.; Cao, Y.; Povinelli, M. L.; Dapkus, P. D.; Zhou, C. GaAs Nanowire Array Solar Cells with Axial p-i-n Junctions. Nano Lett. 2014, 14 (6), 3293-3303.

(6) Saxena, D.; Mokkapati, S.; Parkinson, P.; Jiang, N.; Gao, Q.; Tan, H. H.; Jagadish, C. Optically pumped room-temperature GaAs nanowire lasers. Nat. Photonics 2013, 7, 963.

(7) Miao, X.; Chabak, K.; Zhang, C.; Mohseni, P. K.; Walker, D.; Li, X. High-Speed Planar GaAs Nanowire Arrays with fmax $>75 \mathrm{GHz}$ by Wafer-Scale Bottom-up Growth. Nano Lett. 2015, 15 (5), 27802786.

(8) Joyce, H. J.; Gao, Q.; Hoe Tan, H.; Jagadish, C.; Kim, Y.; Zou, J.; Smith, L. M.; Jackson, H. E.; Yarrison-Rice, J. M.; Parkinson, P.; Johnston, M. B. III-V semiconductor nanowires for optoelectronic device applications. Prog. Quantum Electron. 2011, 35 (2-3), 23-75.

(9) Iadonisi, G.; Cantele, G.; Chiofalo, M. L. Introduction to Solid State Physics and Crystalline Nanostructures; Springer: Milan, 2014.

(10) Wallentin, J.; Mergenthaler, K.; Ek, M.; Wallenberg, L. R.; Samuelson, L.; Deppert, K.; Pistol, M.-E.; Borgström, M. T. Probing the Wurtzite Conduction Band Structure Using State Filling in Highly Doped InP Nanowires. Nano Lett. 2011, 11 (6), 2286-2290.

(11) Allen, J. E.; Perea, D. E.; Hemesath, E. R.; Lauhon, L. J. Nonuniform Nanowire Doping Profiles Revealed by Quantitative Scanning Photocurrent Microscopy. Adv. Mater. 2009, 21 (30), 3067-3072.

(12) Wallentin, J.; Borgström, M. T. Doping of semiconductor nanowires. J. Mater. Res. 2011, 26 (17), 2142-2156.

(13) Dillen, D. C.; Kim, K.; Liu, E.-S.; Tutuc, E. Radial modulation doping in core-shell nanowires. Nat. Nanotechnol. 2014, 9 (2), 116120 .

(14) Kamimura, J.; Bogdanoff, P.; Ramsteiner, M.; Corfdir, P.; Feix, F.; Geelhaar, L.; Riechert, H. p-Type Doping of GaN Nanowires Characterized by Photoelectrochemical Measurements. Nano Lett. 2017, 17 (3), 1529-1537.

(15) Gutsche, C.; Regolin, I.; Blekker, K.; Lysov, A.; Prost, W.; Tegude, F. J. Controllable p-type doping of GaAs nanowires during vapor-liquid-solid growth. J. Appl. Phys. 2009, 105 (2), 024305.

(16) Regolin, I.; Gutsche, C.; Lysov, A.; Blekker, K.; Li, Z.-A.; Spasova, M.; Prost, W.; Tegude, F. J. Axial pn-junctions formed by MOVPE using DEZn and TESn in vapor-liquid-solid grown GaAs nanowires. J. Cryst. Growth 2011, 315 (1), 143-147.

(17) Burgess, T.; Breuer, S.; Caroff, P.; Wong-Leung, J.; Gao, Q.; Hoe Tan, H.; Jagadish, C. Twinning superlattice formation in GaAs nanowires. ACS Nano 2013, 7 (9), 8105-14.

(18) DeJarld, M.; Teran, A.; Luengo-Kovac, M.; Yan, L.; Moon, E. S.; Beck, S.; Guillen, C.; Sih, V.; Phillips, J.; Milunchick, J. M. The effect of doping on low temperature growth of high quality GaAs nanowires on polycrystalline films. Nanotechnology 2016, 27, 495605.

(19) Tambe, M. J.; Ren, S.; Gradecak, S. Effects of gold diffusion on n-type doping of GaAs nanowires. Nano Lett. 2010, 10 (11), 4584-9.

(20) Salehzadeh, O.; Kavanagh, K. L.; Watkins, S. P. Controlled axial and radial Te-doping of GaAs nanowires. J. Appl. Phys. 2012, 112 (5), 054324 .

(21) Gutsche, C.; Lysov, A.; Regolin, I.; Blekker, K.; Prost, W.; Tegude, F.-J. n-Type Doping of Vapor-Liquid-Solid Grown GaAs Nanowires. Nanoscale Res. Lett. 2010, 6, 65.

(22) Sun, R.; Vainorius, N.; Jacobsson, D.; Pistol, M. E.; Lehmann, S.; Dick, K. A. Sn-seeded GaAs nanowires grown by MOVPE. Nanotechnology 2016, 27 (21), 215603.

(23) Wirths, S.; Weis, K.; Winden, A.; Sladek, K.; Volk, C.; Alagha, S.; Weirich, T. E.; von der Ahe, M.; Hardtdegen, H.; Lüth, H.; Demarina, N.; Grützmacher, D.; SchäPers, T. Effect of Si-doping on 
InAs nanowire transport and morphology. J. Appl. Phys. 2011, 110 (5), 053709.

(24) Borgstrom, M. T.; Norberg, E.; Wickert, P.; Nilsson, H. A.; Tragardh, J.; Dick, K. A.; Statkute, G.; Ramvall, P.; Deppert, K.; Samuelson, L. Precursor evaluation for in situ InP nanowire doping. Nanotechnology 2008, 19 (44), 445602.

(25) Wallentin, J.; Ek, M.; Wallenberg, L. R.; Samuelson, L.; Deppert, K.; Borgström, M. T. Changes in Contact Angle of Seed Particle Correlated with Increased Zincblende Formation in Doped InP Nanowires. Nano Lett. 2010, 10 (12), 4807-4812.

(26) Otnes, G.; Heurlin, M.; Zeng, X.; Borgstrom, M. T. InxGa1-xP Nanowire Growth Dynamics Strongly Affected by Doping Using Diethylzinc. Nano Lett. 2017, 17 (2), 702-707.

(27) Yuan, X.; Caroff, P.; Wong-Leung, J.; Fu, L.; Tan, H. H.; Jagadish, C. Tunable Polarity in a III-V Nanowire by Droplet Wetting and Surface Energy Engineering. Adv. Mater. 2015, 27 (40), 60966103.

(28) Yang, B.; Liu, B.; Wang, Y.; Zhuang, H.; Liu, Q.; Yuan, F.; Jiang, X. Zn-dopant dependent defect evolution in GaN nanowires. Nanoscale 2015, 7 (39), 16237-16245.

(29) Algra, R. E.; Verheijen, M. A.; Borgstrom, M. T.; Feiner, L.-F.; Immink, G.; van Enckevort, W. J. P.; Vlieg, E.; Bakkers, E. P. A. M. Twinning superlattices in indium phosphide nanowires. Nature 2008, 456 (7220), 369-372.

(30) Singh, A. K.; Kumar, V.; Note, R.; Kawazoe, Y. Effects of Morphology and Doping on the Electronic and Structural Properties of Hydrogenated Silicon Nanowires. Nano Lett. 2006, 6 (5), 920925.

(31) Gudiksen, M. S.; Lauhon, L. J.; Wang, J.; Smith, D. C.; Lieber, C. M. Growth of nanowire superlattice structures for nanoscale photonics and electronics. Nature 2002, 415, 617.

(32) Han, N.; Hou, J. J.; Wang, F.; Yip, S.; Yen, Y.-T.; Yang, Z.-x.; Dong, G.; Hung, T.; Chueh, Y.-L.; Ho, J. C. GaAs Nanowires: From Manipulation of Defect Formation to Controllable Electronic Transport Properties. ACS Nano 2013, 7 (10), 9138-9146.

(33) Dayeh, S. A.; Yu, E. T.; Wang, D. III-V Nanowire Growth Mechanism: V/III Ratio and Temperature Effects. Nano Lett. 2007, 7 (8), 2486-2490.

(34) Dayeh, S. A.; Yu, E. T.; Wang, D. Surface Diffusion and Substrate-Nanowire Adatom Exchange in InAs Nanowire Growth. Nano Lett. 2009, 9 (5), 1967-1972.

(35) Zou, J.; Paladugu, M.; Wang, H.; Auchterlonie, G. J.; Guo, Y.N.; Kim, Y.; Gao, Q.; Joyce, H. J.; Tan, H. H.; Jagadish, C. Growth Mechanism of Truncated Triangular III-V Nanowires. Small 2007, 3 (3), 389-393.

(36) Sun, W.; Guo, Y.-N.; Xu, H.-Y.; Liao, Z.-M.; Gao, Q.; Tan, H. H.; Jagadish, C.; Zou, J. Unequal P Distribution in Nanowires and the Planar Layer during GaAsP Growth on GaAs $\{111\}$ B by MetalOrganic Chemical Vapor Deposition. J. Phys. Chem. C 2013, 117 (37), 19234-19238.

(37) Larsen, C. A.; Buchan, N. I.; Li, S. H.; Stringfellow, G. B. Decomposition mechanisms of trimethylgallium. J. Cryst. Growth 1990, 102 (1), 103-116.

(38) Kawaguchi, Y.; Nakashima, K. Sn doping for InP and InGaAs grown by metalorganic molecular beam epitaxy using tetraethyltin. $J$. Cryst. Growth 1989, 95 (1-4), 181-184.

(39) Lee, P. W.; Omstead, T. R.; McKenna, D. R.; Jensen, K. F. In situ mass spectroscopy and thermogravimetric studies of GaAs MOCVD gas phase and surface reactions. J. Cryst. Growth 1987, 85 (1), 165-174.

(40) Kim, Y. S.; Won, Y. S.; Hagelin-Weaver, H.; Omenetto, N.; Anderson, T. Homogeneous Decomposition Mechanisms of Diethylzinc by Raman Spectroscopy and Quantum Chemical Calculations. J. Phys. Chem. A 2008, 112 (18), 4246-4253.

(41) Metaferia, W.; Sivakumar, S.; Persson, A. R; Geijselaers, I.; Wallenberg, L R.; Deppert, K.; Samuelson, L.; Magnusson, M. H ntype doping and morphology of GaAs nanowires in Aerotaxy. Nanotechnology 2018, 29, 285601.
(42) Dayeh, S. A.; Picraux, S. T. Direct Observation of Nanoscale Size Effects in Ge Semiconductor Nanowire Growth. Nano Lett. 2010, 10 (10), 4032-4039.

(43) Zhang, Z.; Lu, Z.; Xu, H.; Chen, P.; Lu, W.; Zou, J. Structure and quality controlled growth of InAs nanowires through catalyst engineering. Nano Res. 2014, 7 (11), 1640-1649.

(44) Zannier, V.; Rossi, F.; Dubrovskii, V. G.; Ercolani, D.; Battiato, S.; Sorba, L. Nanoparticle Stability in Axial InAs-InP Nanowire Heterostructures with Atomically Sharp Interfaces. Nano Lett. 2018, $18,167-174$.

(45) Johansson, J.; Karlsson, L. S.; Dick, K. A.; Bolinsson, J.; Wacaser, B. A.; Deppert, K.; Samuelson, L. Effects of Supersaturation on the Crystal Structure of Gold Seeded III-V Nanowires. Cryst. Growth Des. 2009, 9 (2), 766-773.

(46) Gao, H.; Lysevych, M.; Tan, H. H.; Jagadish, C.; Zou, J. The effect of $\mathrm{Sn}$ addition on GaAs nanowire grown by vapor-liquid-solid growth mechanism. Nanotechnology 2018, 29, 465601.

(47) Lin, X. W.; Liliental Weber, Z.; Washburn, J.; Weber, E. R.; Sasaki, A.; Wakahara, A.; Hasegawa, T. Ge/Si heterostructures grown by $\mathrm{Sn}$ surfactant mediated molecular beam epitaxy. J. Vac. Sci. Technol., B: Microelectron. Process. Phenom. 1995, 13 (4), 1805-1809.

(48) Persson, A. R.; Metaferia, W.; Sivakumar, S.; Samuelson, L.; Magnusson, M. H.; Wallenberg, R. Electron Tomography Reveals the Droplet Covered Surface Structure of Nanowires Grown by Aerotaxy. Small 2018, 14, No. 1801285.

(49) Zhou, C.; Zheng, K.; Liao, Z. M.; Chen, P. P.; Lu, W.; Zou, J. Phase purification of GaAs nanowires by prolonging the growth duration in MBE. J. Mater. Chem. C 2017, 5 (21), 5257-5262.

(50) Joyce, H. J.; Gao, Q.; Tan, H. H.; Jagadish, C.; Kim, Y.; Zhang, X.; Guo, Y.; Zou, J. Twin-free uniform epitaxial GaAs nanowires grown by a two-temperature process. Nano Lett. 2007, 7 (4), 921-6.

(51) Joyce, H. J.; Wong-Leung, J.; Gao, Q.; Tan, H. H.; Jagadish, C. Phase Perfection in Zinc Blende and Wurtzite III-V Nanowires Using Basic Growth Parameters. Nano Lett. 2010, 10 (3), 908-915.

(52) Joyce, H. J.; Gao, Q.; Wong-Leung, J.; Kim, Y.; Tan, H. H.; Jagadish, C. Tailoring GaAs, InAs, and InGaAs Nanowires for Optoelectronic Device Applications. IEEE J. Sel. Top. Quantum Electron. 2011, 17 (4), 766-778.

(53) Givargizov, E. I. Fundamental aspects of VLS growth. J. Cryst. Growth 1975, 31, 20-30.

(54) Ek, M.; Filler, M. A. Atomic-Scale Choreography of VaporLiquid-Solid Nanowire Growth. Acc. Chem. Res. 2018, 51 (1), 118126. 